\title{
Regarding “Amplatzer Vascular Plugs Versus Coils for Embolization of Pulmonary Arteriovenous Malformations in Patients with Hereditary Hemorrhagic Telangiectasia"
}

\author{
Zhongzhi Jia ${ }^{1} \cdot$ Shaoqin $\mathrm{Li}^{1} \cdot$ Guomin Jiang ${ }^{1}$
}

Received: 13 July 2016/Accepted: 1 August 2016/Published online: 8 August 2016

(C) Springer Science+Business Media New York and the Cardiovascular and Interventional Radiological Society of Europe (CIRSE) 2016

To the Editor,

We read with great interest the recent article by Tau et al. [1]. The authors concluded that the use of Amplatzer plugs for the embolization of pulmonary arteriovenous malformations (PAVMs) in patients with hemorrhagic telangiectasia is associated with a significantly lower rate of recanalization of feeding vessels than coils. However, we would like to elaborate on the patient selection of this study.

Firstly, there were a total of 36 patients who received embolization of PAVMs; however, only 16 patients were available for imaging scans and were included in this study. The authors did not clarify why the other 20 patients were not available for imaging scans and how many of them used coils or Amplatzer plugs for the embolization of PAVMs. Secondly, $93.4 \%$ (15/16) patients were proved recanalization of feeding vessels by intrapulmonary angiograms; however, the authors did not explain why those patients underwent intrapulmonary angiograms. We are interested in knowing why those patients underwent intrapulmonary angiograms but CT arteriography. All of which may bias the results.

Acknowledgments No funding was obtained for this study.

Compliance with Ethical Standards

Conflicts of interest Official statements (The No. 2 People's Hospital of Changzhou, Nanjing Medical University), Zhongzhi Jia, Shaoqin Li and Guomin Jiang has indicate that they have no potential conflicts of interest.

\section{Reference}

1. Tau N, Atar E, Mei-Zahav M, et al. Amplatzer vascular plugs versus coils for embolization of pulmonary arteriovenous malformations in patients with hereditary hemorrhagic telangiectasia. Cardiovasc Intervent Radiol. 2016;39(8):1110-4.
Guomin Jiang

747094035@qq.com

1 Department of Interventional Radiology, No. 2 People's Hospital of Changzhou, Nanjing Medical University, Xing long road 29\#, Chang Zhou 213003, Jiangsu province, China 